\title{
Modélisation d'un écoulement diphasique dans un faisceau de tubes par le code CAFCA : application aux générateurs de vapeur des centrales PWR et aux assemblages de réacteurs à neutrons rapides.
}

\author{
Construction of a model for two-phase flow in a tube \\ bundle using the CAFCA system : application to \\ steam generators of $P W R$ power stations and \\ assemblies for fast breeder reactors.
}

\author{
J. Olive, C. Siméon et D. Tincq
}

Electricité de France

Direction des Etudes et Recherches

Service Réacteurs nucléaires et échangeurs, Chatou

\begin{tabular}{|c|c|c|c|c|c|}
\hline & & & & & \\
\hline Symbole & Désignation & Unité & Symbole & Désignation & Unité \\
\hline $\begin{array}{l}g \\
h \\
P \\
Q \\
R \\
S\end{array}$ & $\begin{array}{l}\text { diffusivité thermique turbulente } \\
\text { chaleur massique du fluide pri- } \\
\text { maire } \\
\text { puissance dégagée par unité de } \\
\text { volume } \\
\text { force d'intéraction volumique } \\
\text { suivant la direction } j \\
\text { accélération gravitationnelle } \\
\text { enthalpie massique secondaire } \\
\text { pression secondaire } \\
\text { tenseur de résistance de l'écoule- } \\
\text { ment (tenseur du } 2^{c} \text { ordre) } \\
\text { résistance thermique } \\
\text { surface d'échange thermique vo- } \\
\text { lumique }\end{array}$ & $\begin{array}{r}\mathrm{m}^{2} / \mathrm{s} \\
\mathrm{J} /(\mathrm{kg} \cdot \mathrm{K}) \\
\mathrm{W} / \mathrm{m}^{3} \\
\mathrm{~N} / \mathrm{m}^{3} \\
\mathrm{~m} / \mathrm{s}^{2} \\
\mathrm{~J} / \mathrm{kg} \\
\mathrm{Pa} \\
\mathrm{s}^{-1} \\
\mathrm{~m}^{2} \cdot \mathrm{K} / \mathrm{W} \\
\mathrm{m}^{2} / \mathrm{m}^{3}\end{array}$ & $\begin{array}{l}t \\
T \\
T \\
V \\
V_{p} \\
X \\
\alpha \\
\gamma \\
\varepsilon \\
\varepsilon_{p} \\
v \\
\rho \\
\rho_{p}\end{array}$ & $\begin{array}{l}\text { temps } \\
\text { température secondaire } \\
\text { température primare } \\
\text { vitesse secondaire } \\
\text { vitesse primaire } \\
\text { titre thermodynamique du fluide } \\
\text { secondaire } \\
\text { taux de vide } \\
\text { glissement entre phases } \\
\text { porosité côté secondaire } \\
\text { porosité côté primaire } \\
\text { viscosité cinématique turbulente } \\
\text { masse volumique secondaire } \\
\text { masse volumique primaire }\end{array}$ & $\begin{array}{r}{ }^{\circ} \mathrm{C} \\
{ }^{\circ} \mathrm{C} \\
\mathrm{m} / \mathrm{s} \\
\mathrm{m} / \mathrm{s} \\
\\
- \\
- \\
- \\
- \\
- \\
\mathrm{m}^{2} / \mathrm{s} \\
\mathrm{kg} / \mathrm{m}^{3} \\
\mathrm{~kg} / \mathrm{m}^{3}\end{array}$ \\
\hline
\end{tabular}

De nombreux appareils tels que les générateurs de vapeur, les échangeurs de la filière à eau légère, les assemblages combustibles de la filière à neutrons rapides soulèvent des problèmes qui sont étudiés aussi bien de façon théorique qu'expérimentale.

En général, la compréhension des phénomènes passe par la connaissance des écoulements et des distributions de température dans ces appareils. L'utilisation des codes multidimensionnels peut apporter une contribution importante dans la réalisation de cet objectif.

C'est pourquoi le département TTA (Transferts thermiques et aérodynamique) de la Direction des Etudes et Recherches d'EDF a décidé de développer le code

CAFCA permettant le calcul des écoulements en simple ou double phase et du transfert thermique en deux ou trois dimensions, dans un milieu comportant un grand nombre d'obstacles répartis [11], [13].

On décrit brièvement la méthode de calcul d'écoulement à travers un faisceau de tubes puis on présente deux applications du code :

- la première concerne le calcul d'écoulements diphasiques dans la partie basse d'un générateur de vapeur PWR; - la deuxième concerne le calcul d'un écoulement monophasique dans un assemblage de réacteur à neutrons rapides partiellement bouché. 


\section{Description du code}

\subsection{Equations de base}

L'adaptation des méthodes numériques de résolution des équations de Navier-Stokes tridimensionnelles aux écoulements diphasiques dans des géométries complexes telles que des faisceaux de tubes nécessite des hypothèses simplificatrices prenant en compte la nature du fluide et les conditions aux limites.

\subsubsection{Homogénéisation du faisceau de tubes}

La prise en compte rigoureuse des interfaces solide/fluide conduirait à un système d'équations beaucoup trop complexe à résoudre lorsque le nombre de tubes est supérieur à quelques unités. Aussi, on considère le faisceau de tubes comme un milieu poreux. Les équations de conservation sont moyennées sur de petits volumes de contrôle entourant chaque point (figure 1).

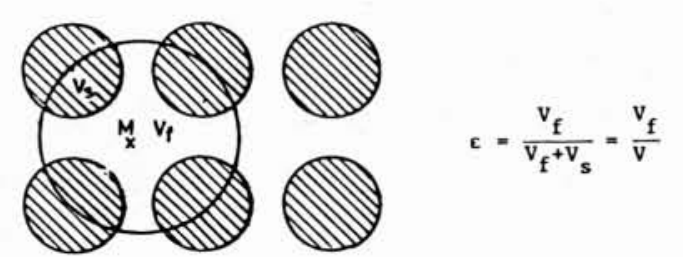

Figure 1. - Exemple de volume d'intégration V.

La proportion de fluide dans chaque volume est la porosité $\varepsilon$ définie par :

$$
\varepsilon=\frac{\text { volume occupé par le fluide }}{\text { volume total }}=\frac{V_{f}}{V}
$$

Les détails de la méthode ont été donnés par Hughes et Chen [1]

L'hypothèse n'est valide que si les volumes d'intégration sont plus grands que les cellules thermohydrauliques élémentaires, de telle sorte que $\varepsilon$ soit une fonction continue des variables d'espace.

Cependant, les équations moyennées font intervenir des termes inconnus supplémentaires. Ces termes ne peuvent être calculés rigoureusement que si les valeurs locales sont connues. Certains d'entre eux peuvent être négligés. Les autres doivent être modélisés par des corrélations empiriques.

\subsubsection{Système final d'équations pour un fluide homo- gène}

Les variables principales $V, P$ et $h$ (vitesse, pression et enthalpie) sont des quantités moyennées. Les équations résultantes sont données ci-dessous (des indices répétés dans un terme indiquent une sommation).

Equation de conservation de la masse :

$$
\frac{1}{\varepsilon \rho} \frac{\partial}{\partial X_{i}}\left(\varepsilon \rho V_{i}\right)=0
$$

Equations de conservation de la quantité de mouvement :

$$
\frac{\partial V_{j}}{\partial t}+V_{i} \frac{\partial V_{i}}{\partial X_{i}}=-\frac{1 \partial P}{\rho \partial X_{j}}+g_{j}+\frac{\partial}{\partial X_{i}}\left(v \frac{\partial V_{i}}{\partial X_{i}}\right)+\frac{F_{i}}{\varepsilon \rho}
$$

Equation de conservation de l'enthalpie :

$$
\frac{\partial h}{\partial t}+\frac{1}{\varepsilon \rho} \frac{\partial}{\partial X_{i}}\left(\varepsilon \rho h V_{i}\right)=\frac{1}{\varepsilon \rho} \frac{\partial}{\partial X_{i}}\left(\varepsilon \rho a \frac{\partial}{\partial X_{i}}\right)+\frac{E}{\varepsilon \rho}
$$

La description des conditions aux limites le long dés parois de tubes n'est plus nécessaire. Elle a été remplacée par une autre difficulté : la détermination des termes source $F$ et $E$ et des coefficents de diffusion turbulente $v$ et $a$.

Le terme transitoire dans l'équation de conservation de la masse a été supprimé. Cela n'est pas gênant puisque le code est orienté vers la détermination d'états stationnaires.

L'équation de quantité de mouvement a été simplifiée en utilisant l'équation de conservation de la masse et est donc écrite sous une forme non conservative.

Lorsque le fluide est diphasique, on utilise le modèle homogène avec équilibre thermodynamique. Cependant, un glissement algébrique entre phases peut être introduit dans le calcul de la masse volumique :

$$
\rho=[X+\gamma(1-X)]\left[\frac{X}{\rho_{r}}+\gamma \frac{(1-X)}{\rho_{l}}\right]^{-1}
$$

Le titre $X$ est calculé à partir de l'enthalpie moyenne du fluide et est fonction de la pression. Le glissement $\gamma$ est obtenu à partir de corrélations. Dans le calcul d'écoulements dans les générateurs de vapeur PWR, on utilise la corrélation de Massena [2], utilisée dans de nombreux codes monodimensionnels.

\subsection{Modélisation des termes sources}

\subsubsection{Terme de source de quantité de mouvement $F$}

Il est écrit sous la forme tensorielle générale :

$$
F_{j}=\varepsilon \rho Q_{i j} V_{i}
$$

selon Butterworth [4], $Q_{i j}$ est un tenseur diagonal si les axes de coordonnées sont des directions principales du faisceau de tubes, ce qui est généralement le cas.

Les $Q_{i i}$ sont des termes de résistance. Ils sont déterminés à partir des corrélations de frottement monodimensionnelles obtenues en monophasique et de multiplicateurs diphasiques.

Le terme diagonal correspondant aux écoulements parallèles aux tubes est modélisé grâce à des corrélations obtenues dans des tubes verticaux.

Quant aux termes diagonaux correspondant aux écoulements transversaux, ils sont modélisés par l'intermédiaire :

- d'un coefficient de perte de charge par unité de longueur en monophasique fourni par les résultats de Boissier [5]; 
- d'un multiplicateur prenant en compte l'angle d'attaque du faisceau par le fluide dans un plan transversal et donné par les expériences de Kazakevic [6];

- d'un multiplicateur diphasique pour lequel peu de résultats existent en écoulements transversaux dans des faisceaux de tubes [7].

\subsubsection{Terme de source d'énergie $E$}

Dans le cas où le flux n'est pas imposé, il est donné par la loi générale de transfert de chaleur :

$$
E=\frac{S}{R}\left(T_{p}-T\right)
$$

La température primaire $T_{p}$ est calculée à partir de l'équation de conservation de l'énergie du fluide primaire :

$$
\frac{\partial T_{p}}{\partial t}+\frac{\partial}{\partial X_{i}}\left(T_{p} V_{p i}\right)=-\frac{E}{\varepsilon_{p} C_{p} \rho_{p}}
$$

$\rho_{p}$ est supposée constante et le champ de vitesse $V_{p}$ est connu a priori.

Pour la résistance thermique de convection entre le fluide primaire et la paroi intérieure des tubes, on utilise la corrélation de Dittus et Boelter [8]. Pour la résistance de convection entre le fluide secondaire monophasique et la paroi externe des tubes, on utilise à nouveau des corrélations obtenues par Boissier [5] pour des écoulements transverses, par Dittus-Boelter pour des écoulements longitudinaux et une méthode d'interpolation pour des écoulements obliques due à Butterworth [9]. Pour les écoulements secondaires diphasiques, une corrélation de Jens et Lottes modifiée (utilisée pour les calculs monodimensionnels de générateur de vapeur) est utilisée.

\subsubsection{Coefficients de diffusion turbulente}

En raison du manque de données pour des écoulements diphasiques dans des faisceaux de tubes, ils sont généralement pris égaux à zéro.

\subsection{Méthode numérique}

La méthode numérique, utilisée dans CAFCA, a été développée par Hughes et Chen [1] sur la base de la méthode MAC modifiée par Hirt et Cook [10]. Seuls les principes généraux sont décrits ici.

\subsubsection{Maillage en différences finies}

Le maillage utilisé est cartésien. Les variables scalaires sont définies au centre de la maille élémentaire. La composante de la vitesse suivant la direction $i$ est définie au centre de la face normale à cette direction (figure 2 ). Une méthode de décentrement variable peut être utilisée pour discrétiser les termes de convection.

\subsubsection{Méthode de résolution}

Le code calcule un pseudo-transitoire par pas de temps successifs jusqu'à atteindre l'état stationnaire. Chaque étape de temps se subdivise en trois phases:

a) Calcul explicite des nouvelles valeurs de la température primaire, de l'enthalpie et de la masse volumique en utilisant les formes discrétisées des équations (3), (4), (6) et (7).

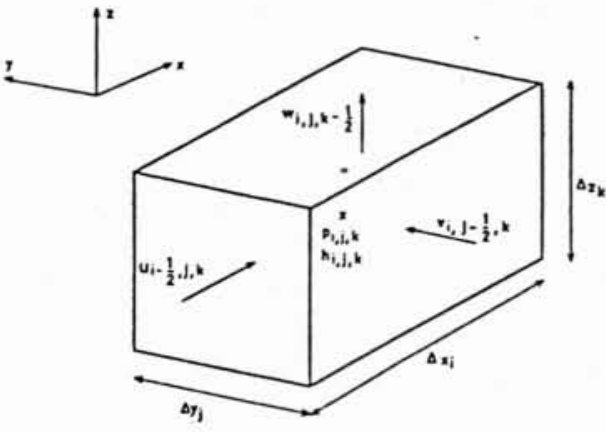

Figure 2. - Localisation des principales variables dans la cellule de calcul élémentaire.

b) Calcul explicite des vitesses avancées en temps, en utilisant la forme discrétisée des équations (2) et (5), la pression étant évaluée à l'ancien pas de temps.

c) Calcul implicite des nouvelles pressions et vitesses par une méthode itérative utilisant les équations (1) et (2). A chaque itération, la pression et les composantes de la vitesse dans chaque cellule sont corrigées par une quantité proportionnelle à l'accumulation de masse dans la cellule.

Le pas de temps, de même que le paramètre de relaxation et le critère de convergence de ta méthode itérative sont optimisés de façon à accélérer la convergence globale vers l'état stationnaire sans violer le critère de stabilité.

\subsubsection{Conditions aux limites}

Elles sont fournies en utilisant des cellules fictives autour du domaine de calcul. Elles permettent de simuler des parois imperméables, des plans de symétrie, des écoulements entrants ou sortants.

\section{Application} à la partie basse d'un générateur de vapeur PWR

\subsection{Introduction}

La région située juste au-dessus de la plaque tubulaire des générateurs de vapeur est très exposée aux risques de corrosion des tubes. Cela est lié à l'existence d'une zone de faibles vitesses qui favorise l'accumulation des dépôts ferritiques.

Une simulation de cette zone par CAFCA-3 a été réalisée afin de mieux comprendre ces phénomènes de dépôts et de comparer les résultats numériques à certains résultats expérimentaux.

\subsection{Maillage et conditions aux limites}

La région simulée est un cylindre délimité latéralement par la virole intérieure, par la plaque tubulaire à la partie inférieure et par la troisième plaque entretoise à la partie 
supérieure (figure 3). Les conditions aux limites d'entrée sont les profils de vitesse et d'enthalpie à travers la fenêtre d'entrée qui sont connus grâce aux résultats expérimentaux. A la sortie supérieure, on impose une condition de pression uniforme.

Les plaques entretoises sont simulées par des sources de quantité de mouvement de grande intensité appliquées localement, modélisées par un tenseur de résistance diagonal.

\subsection{Résulats du calcul}

On présente les résultats d'un calcul effectué aux conditions nominales de fonctionnement du générateur de vapeur.

La figure 4 montre le champ de vitesse et la figure 5 les lignes d'isotitre dans le plan vertical de symétrie (qui n'est pas un plan de symétrie de l'écoulement à cause des conditions aux limites non symétriques).

La figure 6 montre les lignes d'égales vitesses et la figure 7 les lignes d'isotitre juste au-dessus de la plaque tubulaire : la zone de basse vitesse $(\leqslant 0,29 \mathrm{~m} / \mathrm{s})$ du côté de la jambe chaude a une étendue limitée qui coïncide avec la zone de vaporisation.

Les résultats des calculs ont été comparés à deux types de données expérimentales :

- la zone-d'accumulation des dépôts, illustrée sur les figures 6 et 7 , est détectée par courants de Foucault sur tous les générateurs de vapeur de ce type. Elle se situe dans le domaine de vaporisation et coïncide presque avec la zone de basse vitesse en jambe chaude calculée par le code:

- les températures mesurées le long d'un diamètre perpendiculaire à la rue d'eau. La figure 8 montre qu'il y a un bon accord entre le profil de température calculé par CAFCA-3 et les résultats de mesure.

\section{Application aux assemblages de réacteurs à neutrons rapides}

L'analyse des conséquences de l'obstruction de plusieụrs sous-canaux d'un assemblage est un élément essentiel dans la démarche de sûreté des réacteurs à neutrons rapides.

En vue de cette analyse, un grand nombre d'expériences ont été effectuées aussi bien en France qu'à l'étranger. Le code CAFCA a été utilisé pour interpréter un certain nombre de ces expériences. La comparaison de quelques résultats avec les données expérimentales correspondantes est présentée ici.

\subsection{Bouchage local de 6 sous-canaux dans une grappe de 37 aiguilles}

Une série d'expériences a été effectuée sur une grappe de 37 aiguilles dans laquelle un bouchage de 6 sous-canaux a été introduit. Deux types d'expériences ont été réalisés :

- des expériences hydrauliques, sur grappe non chauffante et en similitude de Reynolds avec la grappe de 37 aiguilles testée en sodium, qui ont permis de mesurer le champ de vitesse (figure 9);

- des expériences en sodium sur une grappe de 37 aiguilles chauffantes durant lesquelles les champs de températures ont été mesurés.

\subsection{1. - Comparaison des champs de vitesses}

Les profils axiaux de vitesses dans différents sous-canaux ont pu être mesurés sur la maquette hydraulique et ont pu être calculés avec une version bidimensionnelle axisymétrique du code CAFCA.

Le domaine de calcul est cylindrique et le milieu est supposé poreux $(\varepsilon=0.335$ ) (figure 10). Le bouchage est supposé cylindrique. Les diamètres du bouchage et du domaine sont tels que les sections de passage du fluide sont les sections réelles, dans toute section droite de l'assemblage.

Les calculs ont été faits avec une vitesse d'entrée de $0,86 \mathrm{~m} / \mathrm{s}$. $(\operatorname{Re}=35000)$.

Sur les figures 11 et 12 , on constate un bon accord entre les calculs et la mesure de vitesse en aval du bouchage : la longueur de la zone de recirculation est correcte mais les vitesses de recirculation calculées sont plus grandes que celles déduites des mesures.

\subsubsection{Comparaison des champs de températures}

Le cas de calcul présenté correspond aux conditions de fonctionnement suivantes :

- nombre de Reynolds à l'entrée : 14000

- température d'entrée : $281^{\circ} \mathrm{C}$

- puissance dissipée : $312 \mathrm{~kW}$.

La figure 13 présente les profils de température calculés dans les couronnes, constituant les limites externes et internes du bouchage. Elles sont comparées aux mesures de température dans les sous-canaux bouchés :

- l'élévation de température en aval du bouchage est correctement calculée;

- la majeure partie des points de mesure se situe entre les deux courbes; toutefois, le calcul prévoit un refroidissement plus rapide des sous-canaux 1 et 2 ;

- la longueur du sillage thermique est correcte.

\subsection{Bouchage de $49 \%$ de la section de passage dans une grappe de 169 aiguilles (type SNR 300)}

Le "Liquid Metal Boiling Working Group " a proposé, au cours de sa $9^{e}$ session, un exercice visant à calculer les conditions thermohydrauliques d'un assemblage partiellement bouché. L'assemblage est du type SNR 300; la section bouchée varie entre $34,4 \%$ et $68,5 \%$ de la section de passage totale.

Les calculs ont été effectués avec les codes CAFCA (EDF), SABRE (UKAEA), ASFRE (PNC) et les résultats ont été publiés [14].

A l'aide du code CAFCA, les calculs n'ont été faits que sur les bouchages centraux couvrant $49,68,5$ et $34,4 \%$ de la section de passage.

Un exemple de résultat, obtenu sur le bouchage de $49 \%$, est donné sur la figure 14. On observe un accord correct entre le champ de température mesuré et calculé.

De forts gradients de vitesse et de température dans la zone de recirculation caractérisent cet exercice : leur 


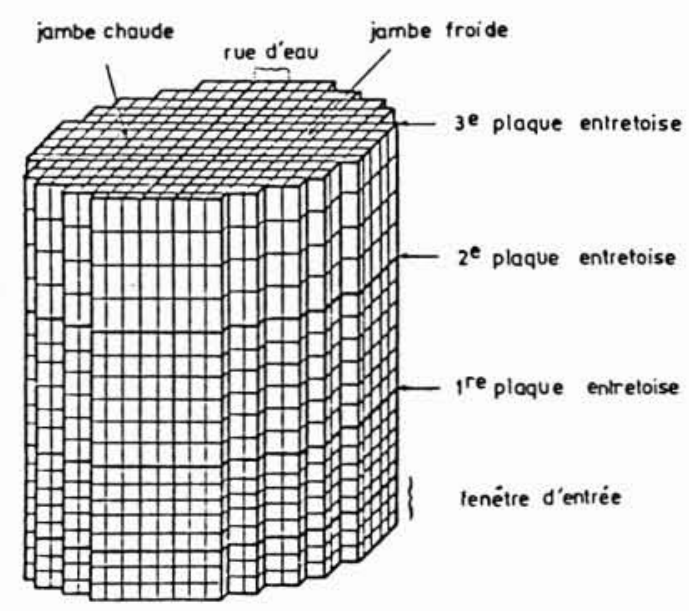

Figure 3. - Maillage utilisé dans un calcul de bas de GV de type 51 .

jambe chaude

jambe froide

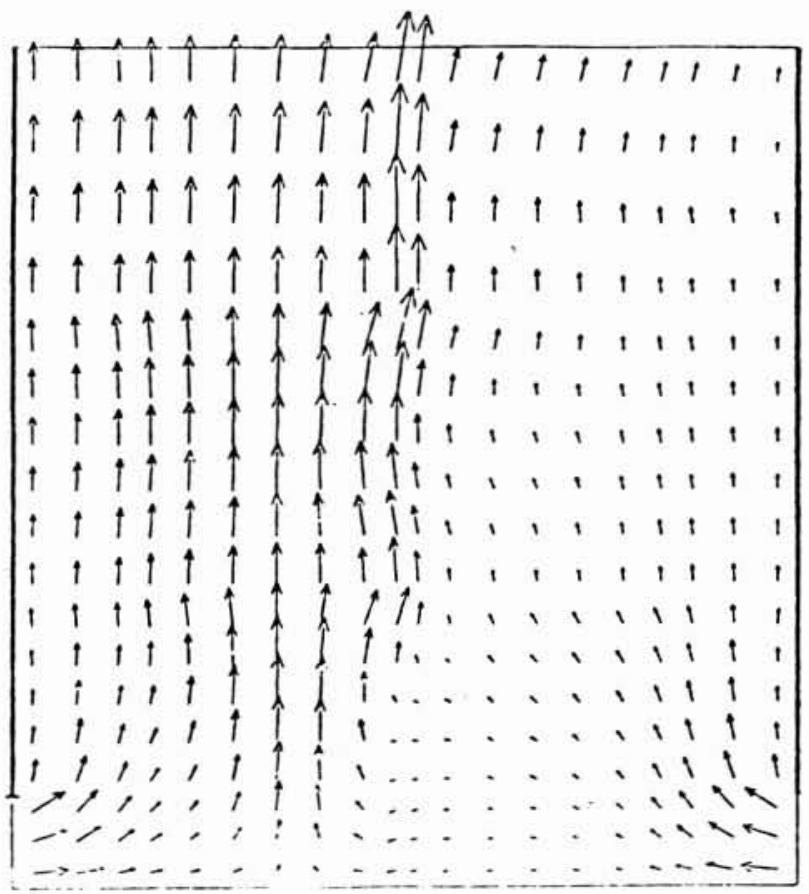

Figure 4. - Vecteurs vitesses dans le plan vertical.

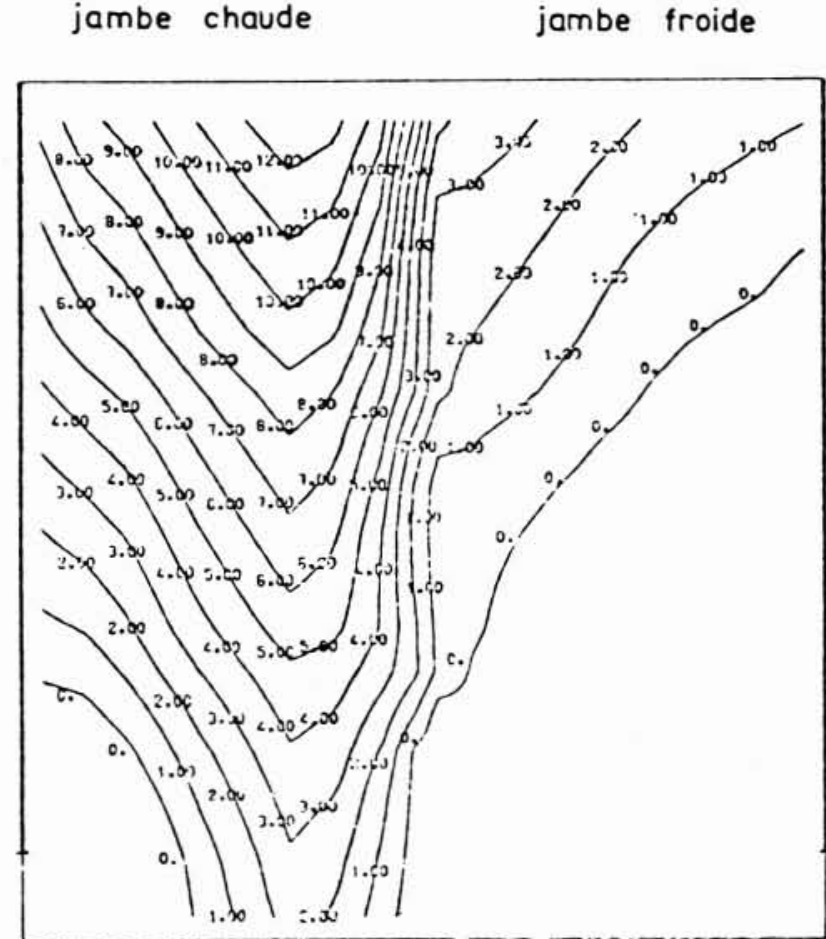

Figure 5. - Lignes d'iso-titre dans le plan vertical de symétrie (valeurs des titres en \%).

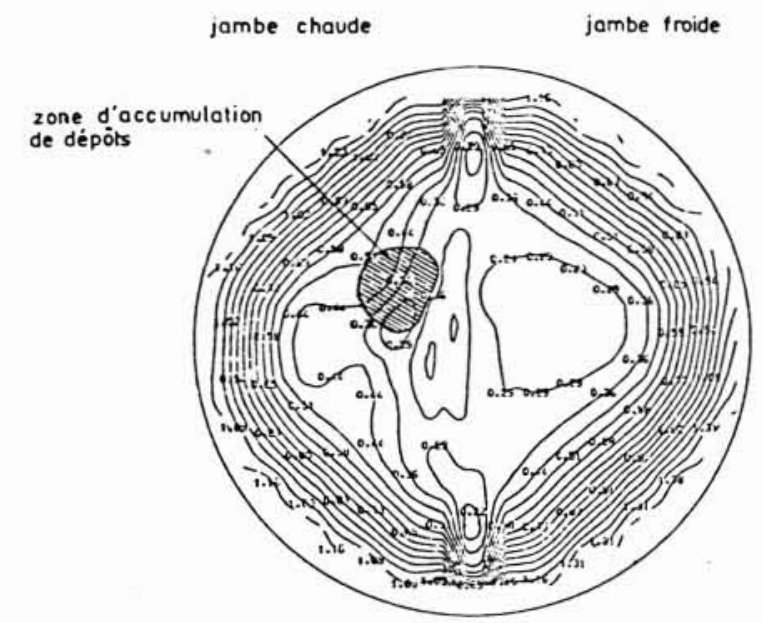

Figure 6. - Lignes d'iso-vitesse sur la plaque tubulaire (en $\mathrm{m} / \mathrm{s}$ ). 


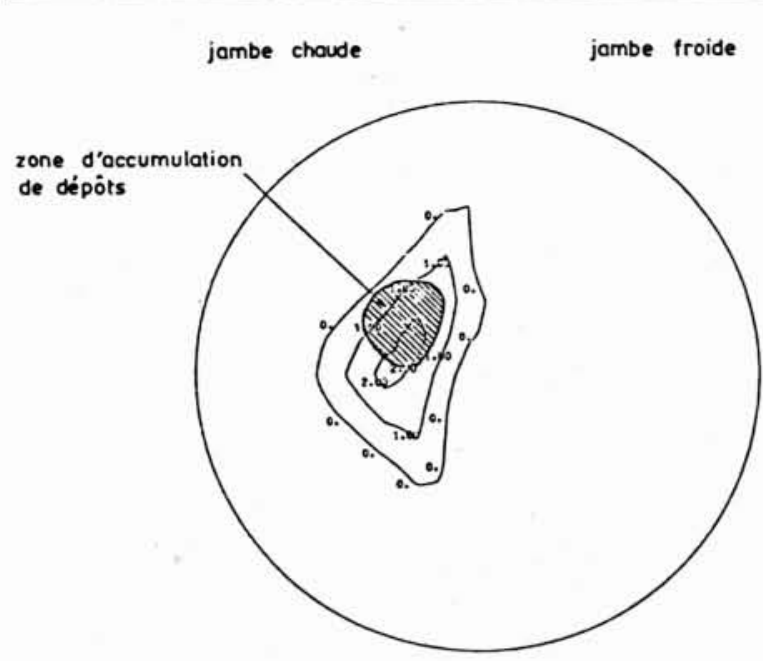

Figure 7. - Lignes d'iso-titre sur la plaque tubulaire (en \%).

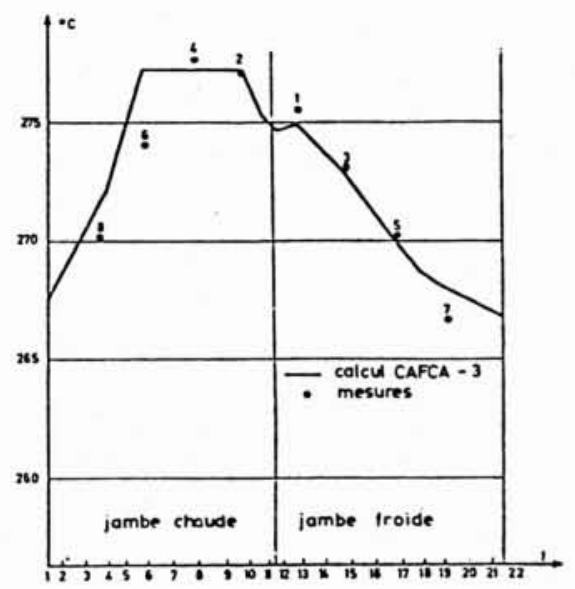

Figure 8. - Profil de température sur la plaque tubulaire.

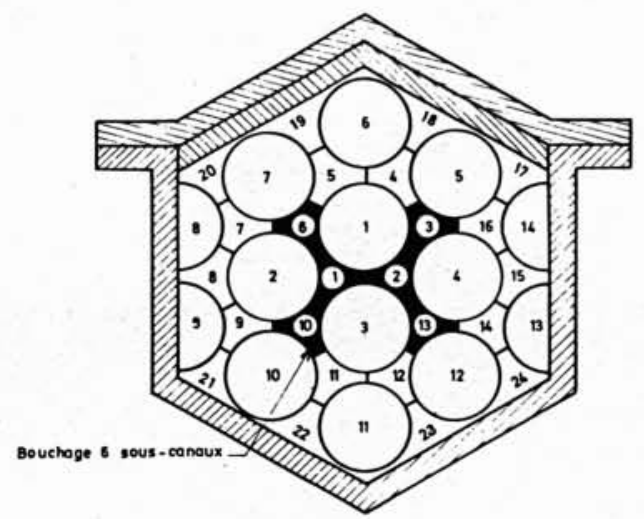

Figure 9. - Coupe de la maquette.

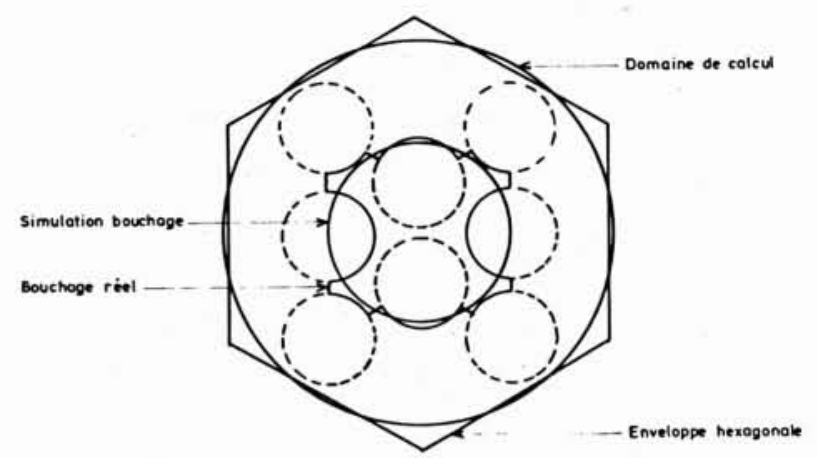

Figure 10. - Domaine de calcul du code CAFCA.

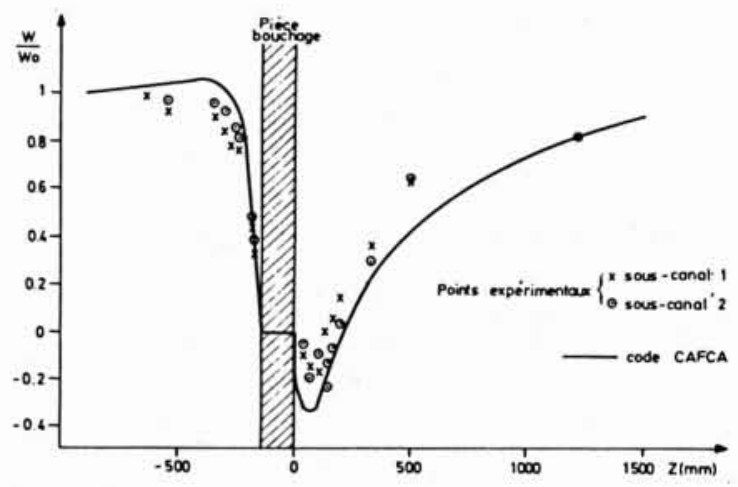

Figure 11. - Bouchage étanche sous-canaux 1 et 2.

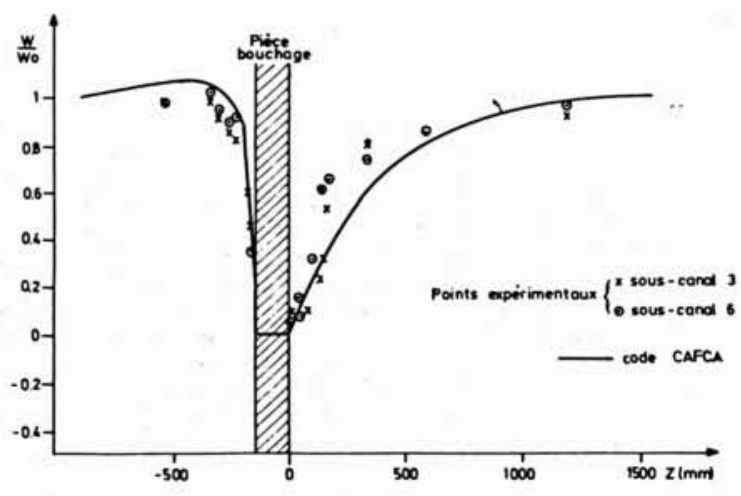

Figure 12. - Bouchage étanche sous-canaux 3 et 6. 


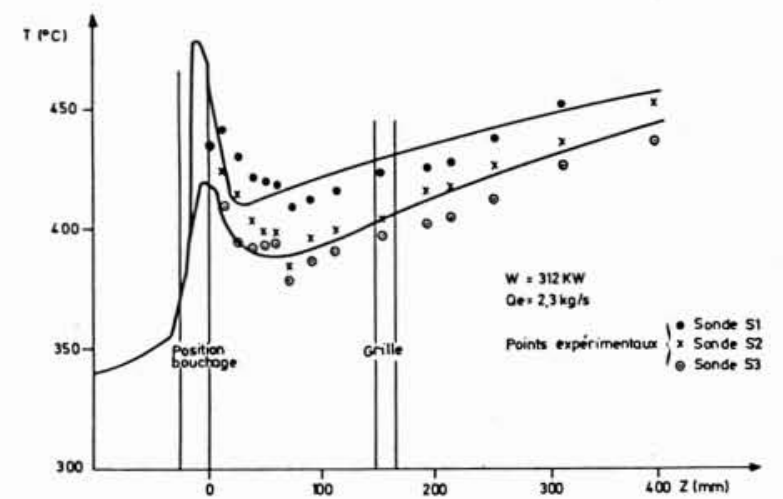

Figure 13. - Profils de température dans les sous-canaux bouchés. $\mathrm{RE}=14000$
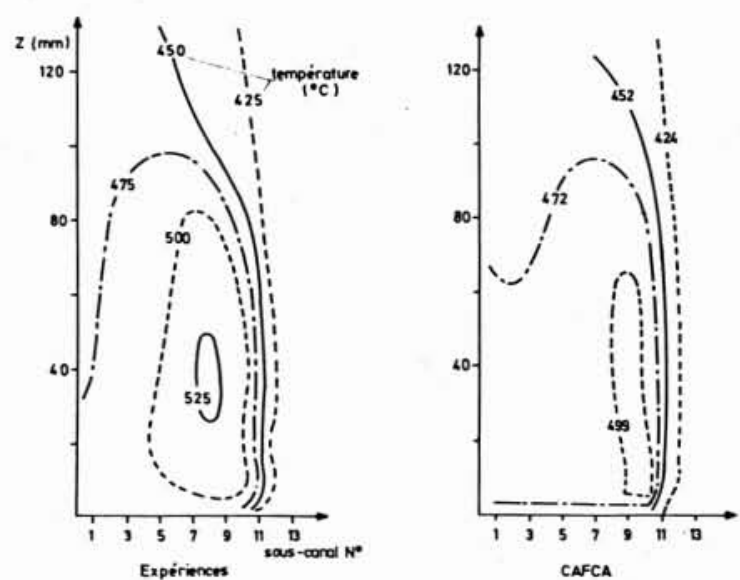

Figure 14. - Calculs effectués dans le cadre de l'exercice du LMBWG - Bouchage central de $49 \%$.

Essai n०6 - Comparaison des distributions de température. D'après [14]. représentation correcte a nécessité une bonne prise en compte des phénomènes de diffusion.

Un soin particulier au niveau de la méthode numérique a permis de diminuer les phénomènes de diffusion parasites, tout en préservant la stabilité du schéma. Des corrélations pour le calcul des coefficients de diffusion sont introduites dans les équations de conservation.

\section{Développements et validations futurs du code}

\subsection{Développements du modèle diphasique}

Le modèle présenté n'est valable pour calculer des écoulements diphasiques que si les vitesses des deux phases sont colinéaires. Dans le cas où ces vitesses ne sont plus parallèles, un vecteur " vitesse relative entre phases " doit être introduit dans les équations de conservation du mélange. Il peut être fourni par une équation vectorielle aux dérivées partielles supplémentaire ou simplement par une corrélation, ce qui permet de conserver un modèle à 3 équations. Un tel développement est en cours.

\subsection{Programme expérimental de validation}

Les corrélations utilisées dans CAFCA sont, en général, des corrélations établies pour des écoulements monodi- mensionnels et ne couvrent pas l'ensemble du domaine d'application du code (écoulement diphasique transversal à un faisceau de tubes, en particulier). Afin d'améliorer les connaissances sur ce sujet, on a entrepris la construction de deux installations expérimentales en écoulement diphasique. La boucle MEDOC, réalisée au Département TTA, utilisera un mélange d'eau liquide et de "Fréon 13 B1 " gazeux et fonctionnera de façon isotherme. La boucle FRIDA, conçue et réalisée en collaboration entre les Départements LNH et TTA, utilisera du "Fréon 114 " sous forme liquide et gazeuse.

\section{Conclusion}

Dans son état de développement actuel, le code CAFCA est un outil opérationnel permettant de déterminer les champs de vitesses et de températures dans un milieu tubulaire tridimensionnel. Les comparaisons expériencescalculs, effectuées jusqu'à maintenant dans des géométries très différentes, sont, en effet, très encourgeantes.

La validation doit être poursuivie afin d'améliorer les modèles physiques (termes sources, diffusion, vitesse relative entre phases) introduits dans CAFCA. En parallèle, le code lui-même sera amélioré par l'utilisation de modèles diphasiques plus élaborés.

\section{Références}

[1] HUGHES E.D., CHEN F.T.W. - Transient, three-dimensional thermal-hydraulic analysis of homogeneous two-phase flows in heat exchangers. AIChE National Heat Transfer Conference. P.W.R. Steam Generators (1977).

[2] MASSENA W.A. - Steam-water pressure drop and critical discharge flow. A digital computer program. HW-65706 Report (1960).
[3] ISHII M. - Thermo-fluid dynamic theory of two-phase flow. Collection de la Direction des Etudes et Recherches d'Electricité de France, Eyrolles - Paris (1975).

[4] BUTTERWORTH D. - The development of a model for three-dimensional flow in tube bundles. Int. J. Heat Mass Transfer, Vol 21, pp. 256-258, (1978). 
[5] BOISSIER A. et al. - Les pertes de charge et le transfert thermique côté gaz dans les échangeurs à tubes lisses à circulation orthogonale. Bulletin de la Direction des Etudes et Recherches d'Electricité de France, série A, n० 2/3 (1971).

[6] IDELC'IK I.E. - Mémento des pertes de charge. Collection du Centre de Recherches et d'Essai de Chatou. Eyrolles, Paris (1969).

[7] ISHIHARA K., PALEN J.W., TABOREK J. - Critical review of correlations for predicting two phase flow pressure drop across tube banks. Heat Transfer Engineering, Vol. I, no 3 - (janvier-mars 1980).

[8] DitTUS F.W., BOELTER L.M.K. - Publications in Engineering, Vol. 2, p. 443 (1930).

[9] BUTTERWORTH D. - A model for heat transfer during three-dimensional flow in tube bundles. HTFS report RS 200 - AERE R8822 (1977).
[10] HIRT C.W., COOK J.L. - Calculating three-dimensional flows around structures and over rough terrain. Journal of Computational Physics, Vol. 10, pp. 324-340 (1972).

[11] SIMEON C. - Calculation of the velocity and temperature field in a fuel pin assembly with the use of the CAFCA code. IMACS " Montreal-82 " congress on "Modeling and Simulation of Nuclear Power Plants ” (1982).

[12] DUKOWICZ J.C., RAMSHAW J.D. - Tensor viscosity method for convection in numerical fluid dynamics. Journal of computational Physics, Vol. 32, pp. $71-79$ (1979).

[13] OLIVE J. - Simulation of three dimensional two-phase flow in a PWR steam generator. IMACS " Montreal-82 " congress on "Modeling and Simulation of Nuclear Power Plants" (1982).

[14] PEPPLER W., HUBER F., DORR B. - Comparaison of benchmark calculations proposed by the LMBWG. 9th Meeting of Liquid Metal Boiling Working Group, Rome, (4-6 juin 1980).

\section{Discussion}

Président : M.H. MONDIN

M. SAGNER. - J'ai 2 questions relatives aux équations du modèle :

a) Est-ce que l'utilisation d'un seul coefficient de porosité volumique implique une hypothèse de milieux poreux isotrope, qui n'est manifestement pas réaliste ?

b) Est-ce que la loi de pertes de charge est laminaire, comme le suggère la formulation des forces exercées par les tubes?

M. Simeon. - Les effets du faisceau de tubes sont pris en compte par la porosité (finalement relativement peu importante), le frottement et le transfert de chaleur (dont les coefficients ont une grande importance).

Pour la porosité, le choix d'une formulation très simplifiée par le terme $\varepsilon$ a été motivé par un souci de simplification, et pour obtenir rapidement un code qui tourne. La première étape dans la mise au point d'un code est numérique et informatique. Ensuite, on s'intéresse à trouver de meilleures corrélations.

Quant au frottement, il est bien proportionnel au carré de la vitesse, et est inspiré des lois de type "BLASIUS». Seule la notation tensorielle utilisée masque un peu cette dépendance par rapport au carré de la vitesse puisqu'un terme de vitesse est passé à l'intérieur du tenseur. D'autre part, on rajoute au niveau des plaques entretoises un tenseur de pertes de charges singulières.

Une étude de sensibilité nous a montré que les résultats sont très sensibles aux coefficients de pertes de charge, en particulier au coefficient de pertes de charge transverse.

Dans le calcul de la résistance thermique entre la paroi des tubes et l'écoulement secondaire monophasique, on utilise deux corrélations, l'une en écoulements obliques, l'autre en écoulements transverses. On les combine quadratiquement pour obtenir la résistance en écoulements obliques.

Ce sont ces coefficients de frottement et de transfert thermique qui rendent compte de la forte anisotropie du milieu.

M. Le Président. - Sur quoi les avez-vous ajustés ?

M. Simeon. - Nos corrélations de pertes de charge transverses proviennent d'expériences réalisées à Chatou et par la Société BERTIN.

Les écoulements dans l'axe des tubes n'ont pas posé de problèmes. Pour les écoulements obliques, nous n'avons pas une très grande confiance dans nos corrélations.

La corrélation d'échange thermique en écoulements transversaux monophasiques provient également de résultats obtenus par M. BOISSIER à Chatou.
M. P. Aujollet. - Prévoyez-vous le développement d'un modèle de transport des boues dans un générateur de vapeur?

M. TINCQ. - La modélisation théorique en est ardue. Des expériences ont lieu à Chatou en écoulement monophasique à froid pour obtenir des corrélations. La mise au point et l'implantation d'un modèle simple de transport et de dép̂ot des boues sont actuellement discutées mais aucune décision n'a été prise pour l'instant à leur sujet.

M. RIGAUDEAU. - Je reviens sur les problèmes de porosité. On peut considérer soit une porosité volumique, soit une porosité surfacique. Il existe un article de Hughes et Chen qui traite le problème mathématique de façon très complète. Ces auteurs ont montré que l'on pourrait ainsi très bien rendre compte des phénomènes liés à la présence d'un faisceau tubulaire, ou même d'un cour.

Jai également une question au sujet de la notion de glissement. Dans un écoulement tridimensionnel, le glissement est vectoriel, alors qu'il est scalaire dans les équations de CAFCA. Cela n'est-il pas génant?

M. TINCQ. - Nous n'utilisons actuellement que trois équations de bilan : masse, quantité de mouvement, énergie et le glissement est effectivement scalaire dans l'état actuel du code.

Nous envisageons d'utiliser une corrélation pour obtenir des vitesses de phases non colinéaires et le code a été modifié dans ce sens.

Jean OLIVE a introduit une corrélation simplifiée de vitesse de montée de bulles. Le code tourne bien et les résultats sont peu sensibles á cette modification. Avec des corrélations plus complexes, le code ne converge pas pour l'instant mais les efforts continuent. Si nous n'arrivons pas à des résultats satisfaisants, nous envisagerons peut-être une formulation à 4 équations, dont 2 équations de quantité de mouvement.

Des vitesses non colinéaires conduisent à un modèle sophistiqué qui ne se justifie que dans le bas du faisceau où les écoulements transverses sont très importants. Elles rendent caduque la définition du titre dynamique habituel et conduisent à définir un titre dynamique fonction non seulement du point mais aussi de la direction.

Néanmoins, dans les corrélations de perte de charge on utilise le titre dynamique défini dans la direction moyenne de l'écoulement. 